
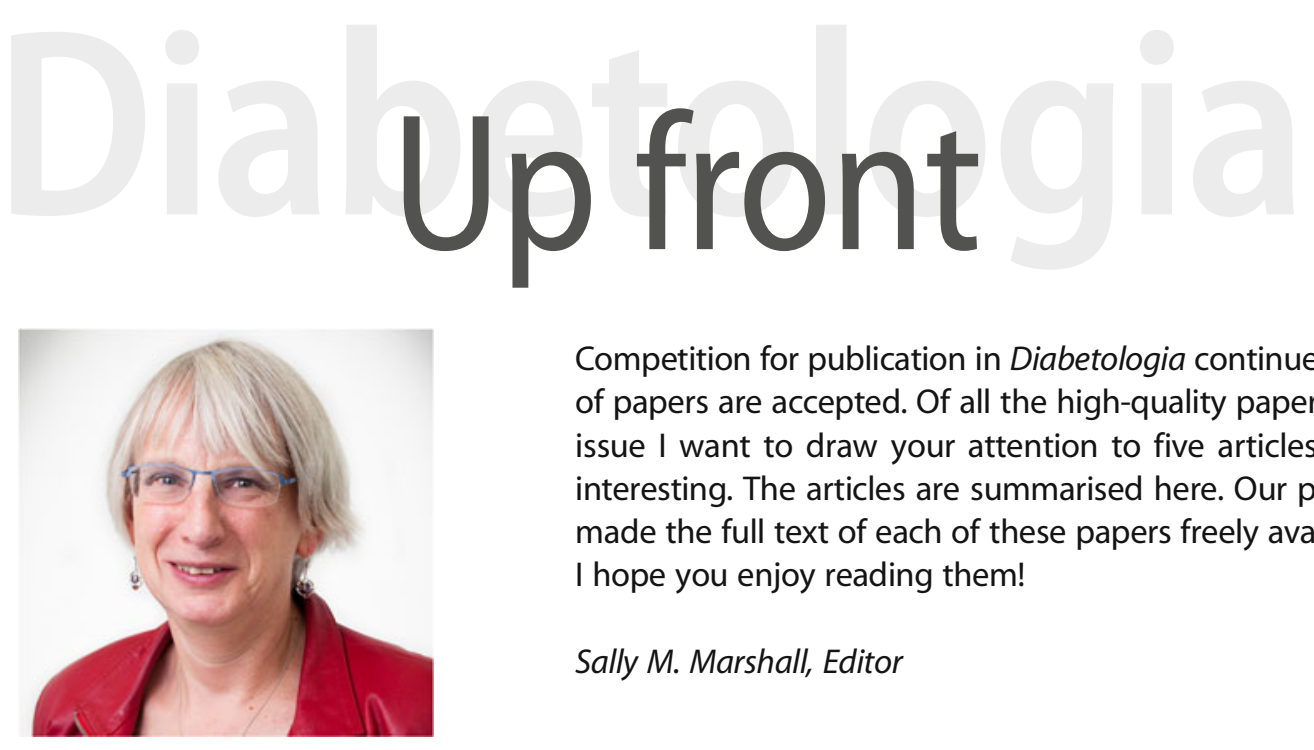

Competition for publication in Diabetologia continues to grow, and less than $20 \%$ of papers are accepted. Of all the high-quality papers that appear in this month's issue I want to draw your attention to five articles that I think are particularly interesting. The articles are summarised here. Our publisher, Springer, has kindly made the full text of each of these papers freely available.

I hope you enjoy reading them!

Sally M. Marshall, Editor

\section{Adipose tissue macrophages: going off track during obesity}

Lily Boutens, Rinke Stienstra

Tissue macrophages are key cells of our innate immune system. These cells, including those in the adipose tissue, are assigned several tasks, including immune surveillance, phagocytosis and cytokine production. The precise functional output of a macrophage is determined by a variety of local and systemic signals. In a review in this issue, Lily Boutens and Rinke Stienstra summarise the differences in functional output between adipose tissue macrophages (ATMs) in lean and obese adipose tissue. In addition, potential local and systemic signals that shape ATM function are discussed. Disturbances in the balance between these signals may eventually lead to a dysfunctional macrophage promoting the development of adipose tissue inflammation and insulin resistance in obese individuals. The integration of data on transcription and functions executed will greatly help to identify relevant signals that determine ATM behaviour during obesity and aid the search for targeted interventions in individuals with diabetes.

\section{Microbial transmission from mothers with obesity or diabetes to infants: an innovative opportunity to interrupt a vicious cycle}

Taylor K. Soderborg, Sarah J. Borengasser, Linda A. Barbour, Jacob E. Friedman

The risk factors for obesity and metabolic diseases begin operating in early life (even in utero) and may permanently change the body's structure, physiology and metabolism, leading to an increased lifetime disease risk and/or disease acceleration. A novel possible mechanistic pathway linking maternal obesity and diabetes with childhood disease involves the effects of maternal metabolic status on early microbiome colonisation of the infant gastrointestinal tract. Early dietary exposures affect the infant gut microbiome, which changes rapidly and reproducibly during the first 24 months of life, with long-lasting effects on the host. Maternal obesity is associated with an altered infant gut microbiome; however, the inciting metabolic factors, and mechanistic links to early disease pathways in the infant, need further study. In a review in this issue, Soderborg et al discuss how maternal metabolism impacts the early infant gut microbiome and how this may lay the seeds for obesity and diabetes disease risk and offer important opportunities for early intervention.

\section{Gastric bypass surgery vs intensive lifestyle and medical intervention for type 2 diabetes: the CROSSROADS randomised controlled trial}

David E. Cummings, David E. Arterburn, Emily O. Westbrook, Jessica N. Kuzma, Skye D. Stewart, Chun P. Chan, Steven N. Bock, Jeffrey T. Landers, Mario Kratz, Karen E. Foster-Schubert, David R. Flum

Roux-en-Y gastric bypass (RYGB) causes remission of type 2 diabetes in most cases, in part through weight-independent mechanisms. Hence, there is increasing interest in 'metabolic surgery', i.e. the use of RYGB and related operations primarily 
to treat type 2 diabetes, including among patients not obese enough to qualify for traditional bariatric surgery. In this issue, Cummings et al provide Level- 1 evidence supporting that concept. They directly compared RYGB with the most intensive supervised lifestyle/medical intervention (with $\geq 45 \mathrm{~min}$ of aerobic exercise, $\geq 5$ days/week) yet tested against surgery in a randomised controlled trial, including in patients with a $\mathrm{BMI}<35 \mathrm{~kg} / \mathrm{m}^{2}$. Although the fitness of the lifestyle/medical patients greatly increased (whereas that of the surgical patients did not) and they showed substantial reductions in weight and blood glucose, at 1 year the rate of diabetes remission $\left(\mathrm{HbA}_{1 \mathrm{c}}<6.0 \%\right.$ [42.1 mmol/mol] off diabetes medications) in the surgery patients was ten times that in the lifestyle/medical group ( $60.0 \%$ vs $5.9 \%, p=0.002)$, and they showed greater reductions of other cardiovascular risk factors and fewer adverse events. This study supports new recommendations to consider metabolic surgery to treat type 2 diabetes in patients with a BMI as low as $30 \mathrm{~kg} / \mathrm{m}^{2}$. This article is the subject of a commentary in this issue by Karl Neff and Carel le Roux.

GLP-1 receptors exist in the parietal cortex, hypothalamus and medulla of human brains and the GLP-1 analogue liraglutide alters brain activity related to highly desirable food cues in individuals with diabetes: a crossover, randomised, placebo-controlled trial

Olivia M. Farr, Michail Sofopoulos, Michael A. Tsoukas, Fadime Dincer, Bindiya Thakkar, Ayse Sahin-Efe, Andreas Filippaios, Jennifer Bowers, Alexandra Srnka, Anna Gavrieli, ByungJoon Ko, Chrysoula Liakou, Nickole Kanyuch, Sofia TseleniBalafouta, Christos S. Mantzoros

Glucagon-like peptide-1 (GLP-1) analogues have gained attention recently because of their ability to cause significant weight loss and are approved for the treatment for obesity and type 2 diabetes. In this issue, Farr et al present the results of their study to determine whether this weightreducing effect stems from actions in the human brain. They report that GLP-1 receptors are present in the human brain, including the cortex. In a crossover, placebo-con- trolled, randomised, double-blinded trial (18 individuals with type 2 diabetes included in analysis), liraglutide, a GLP-1 analogue, given at doses approved for type 2 diabetes, decreased brain activations related to attention and reward signalling in the human brain to highly desirable (high energy or fat) as compared with less desirable food cues. These data indicate that a central mechanism is involved in the effects of liraglutide on metabolism and weight loss. Further studies in larger cohorts are needed to confirm and extend these findings.

\section{Cancer incidence in persons with type 1 diabetes: a five-country study of 9,000 cancers in type 1 diabetic individuals}

Bendix Carstensen, Stephanie H. Read, Søren Friis, Reijo Sund, IImo Keskimäki, Ann-Marie Svensson, Rickard Ljung, Sarah H. Wild, Joannes J. Kerssens, Jessica L. Harding, Dianna J. Magliano, Soffia Gudbjörnsdottir, on behalf of the Diabetes and Cancer Research Consortium

Earlier studies of cancer in individuals with diabetes have not distinguished between diabetes types. As type 1 diabetes constitutes only a small proportion of all diabetes cases $(<10 \%)$, the association between type 1 diabetes and cancer remains unclear. In this issue, Carstensen et al report on an analysis of people with type 1 diabetes who were identified from five nationwide registers. The authors found that type 1 diabetes was associated with an increased risk of cancers of the stomach, liver, pancreas, kidney, ovary and endometrium, whereas the risks of prostate and breast cancer were decreased. Overall, the excess risk was $1 \%$ for men and $7 \%$ for women. Increased risk of cancer among people with type 1 diabetes compared with the general population was highest in the first year following diabetes diagnosis, consistent with earlier cancer detection through increased medical attention. The absence of a dose-response relationship between diabetes duration (and hence duration of insulin use) and cancer incidence indicates that insulin therapy does not contribute substantially to the excess incidence.

All text supplied by the authors. 